\section{User goals-based approach to identify and prioritise the underlying drivers of low concordance with compression hosiery use}

\section{Jerry Hutchinson \\ Hutchinson WoundTech Limited, Neston, UK}

\section{Introduction}

Despite evidence that compression hosiery $(\mathrm{CH})$ is effective in chronic venous disease (CVD), concordance is low. ${ }^{1-3}$ Nonconcordance is associated with poorer outcomes. ${ }^{3}$ There are many underlying reasons for non-concordance some of which also affect the management of type 2 diabetes $^{4}$ and compression in burn injuries. ${ }^{5}$ Factors differ from the perspectives of the patient, product, and health care professional (HCP). All perspectives must be considered to understand the drivers of low concordance. Addressing low concordance from only one perspective is unlikely to generate sustainable improvement in concordance.

\section{Perspectives related to concor- dance}

Many patient-related factors affect concordance. ${ }^{6-10}$ Patients lie on a concordance spectrum. Some adhere closely to the care plan, some quickly remove $\mathrm{CH}$ and never or only occasionally re-apply it. Others lie between these extremes.

Patient beliefs drive concordance. The natural history of CVD is longer than the time scale over which incremental changes can be recognised, perhaps creating the belief that CVD is not serious. Consequently, the patient may believe nothing (or little) will happen if they do not wear $\mathrm{CH},{ }^{10}$ or if they do wear $\mathrm{CH}$. Not wearing $\mathrm{CH}$ may be seen as permissible. The impact passes unnoticed at the level of conscious perception. The patient may struggle with delivery or understanding of complex education provided by the HCP, or believe that it doesn't applies to them. Patients may be elderly; have limited mobility; reduced strength; comorbidities that impair donning/removing $\mathrm{CH}$; may need the assistance which if not available reduces compliance. There may be reasons for non-concordance associated with design that may be patient-related. Low concor- dance may be psychosocial. ${ }^{9}$ The wound itself in a C6 patient may impact concordance. ${ }^{11}$

Patients are likely to want their daily life minimally disrupted. Activities of daily living (ADL) may affect concordance including laundering; replacing/acquiring new $\mathrm{CH} ;{ }^{12}$ confidence related to not being informed about how to replace $\mathrm{CH} ;{ }^{12}$ divergent priorities for the clinical effects of $\mathrm{CH}$, and ADL. Co-morbidities may reduce concordance. The patient may have learned to live with the condition. Family members/carers may see $\mathrm{CH}$ as disruptive, becoming less willing to help. Perhaps the carer is elderly and/or infirm and struggles with donning and removing $\mathrm{CH}$. The patient may start concordant but reduce concordance with experience or changing priorities.

$\mathrm{CH}$ designs may drive non-concordance. The elasticity, stiffness and stretch, which require force to overcome, may be too great. Some $\mathrm{CH}$ open to aid donning and have closures to overcome donning issues. No alteration to the structure/shape/physical properties of the $\mathrm{CH}$ is possible before donning. Performance may degrade once worn and repeatedly laundered, but essentially the properties are fixed. $\mathrm{CH}$ designs may be highly medical but made to look more like consumer products, perhaps reducing respect for $\mathrm{CH}$ making. The comfort of $\mathrm{CH}$ is important. ${ }^{12}$

The HCP expects the condition to be managed by the agreed care plan despite known challenges. The HCP knows that full concordance is unlikely and may identify patients in whom concordance is less likely. The HCP may have history regarding the patient, or general expectations around concordance from the literature and his/her professional experience, or lack knowledge and skills. ${ }^{6-8,13,14}$ Perhaps this uncertainty is communicated to the patient.

\section{Research proposal}

Identifying the drivers of low concordance is highly amenable to research. A productive method of research is to examine the patient's goals (user goals). The use of the term goals instead of needs leads to a different mind-set when pursuing the answers. Focus on user goals rather than the $\mathrm{CH}$ encourages the respondent to consider their drivers and aims. Focusing only on $\mathrm{CH}$ will generate a list of reasons; without user goals it is difficult to determine how to improve concordance/adoption. The two, being highly inter-related, must co-exist.
Correspondence: Jerry Hutchinson, Hutchinson WoundTech Limited, Neston, UK. E-mail: jhutchinson31@gmail.com

Conference presentation: International Compression Club (ICC) Meeting, Paris, 2017.

This work is licensed under a Creative Commons Attribution 4.0 License (by-nc 4.0).

(C) Copyright J. Hutchinson, 2018

Licensee PAGEPress, Italy

Veins and Lymphatics 2018; 7:7624

doi:10.4081/vl.2018.7624

The users include the patient, the HCP, and the patient's carer(s)/family members and all should be involved in any research.

User goals may be Functional, Personal, and Social. Functional goals include managing the condition; reducing oedema; healing the wound; preventing recurrence; reducing pain; minimising need for frequent removal to change or adjust dressings; minimising odour; improving peripheral skin condition. Personal goals include donning and removal; comfort; ability to wear normal clothing; impact on ADL; quality of life; time spent self-caring; laundering. Social goals include outward appearance; social life; group activities; involvement of lay carers; follow up visits to the HCP.

The research is structured to identify the underlying drivers of patient behaviour (goals). First, the steps to achieving goals (Pathway) are identified. The drivers of concordance could lie anywhere on the pathway. With this focus the findings can be stratified, clearly demonstrating where the main drivers of concordance are. Ultimately, user goals are overlaid on the pathway. The pathway is developed by experts, using a structured questionnaire, and validated or developed with patients.

Pathway step one is the HCP consultation including patient history; assessment and diagnosis; define treatment options; patient/carer education; fit with other parts of the care plan; the care plan; set concordance expectations; initiate care plan; repeat visits to the HCP. The second step is implementation of the care plan away from the clinical setting including removing $\mathrm{CH}$ during the day of the consultation; donning and removal on subsequent days (repeated daily); fit with co-morbidities; repeated laundering; $\mathrm{CH}$ replacement as required; removal of new $\mathrm{CH}$ from packaging.

Data collection requires user interviews using open questions considering functional/personal/social goals to identify goals for each pathway component and what makes 
goal achievement easy/difficult. The research should involve $\sim 15$ respondents per $\mathrm{CH}$ type/condition/HCP/carer to identify the drivers of non-concordance with a high degree of confidence. The number would increase for a study in more than one country to account for national differences. Interviews are conducted by an independent, informed interviewer to elicit unfiltered responses. Different users will have different foci for using $\mathrm{CH}$; all will have a bearing on concordance. The research should involve patients using different types of $\mathrm{CH}$ or with different conditions to increase specificity, likely generating different reasons for non-concordance.

This research structure will uncover many stratified reasons for non-concordance and identify main and subordinate drivers. The outcome will inform changes in approach and behaviour by $\mathrm{CH}$ manufacturers, HCPs, patients and carers. Focus on users goals, and understanding why they are or are not being met, will inform new product design, approaches to HCP consultations, enhanced educational materials, and, it is anticipated higher levels of concordance.

\section{References}

1. Arpaia G, Milani M, Addeo R, et al. Clinical validation of a specially sized class II compression knee-sock for the prevention of recurrent ulcers in patients with chronic venous stasis (CEAP 5). Int Angiol 2008;27:507-11.

2. Palfreyman SJ, Michaels JA. A systematic review of compression hosiery for uncomplicated varicose veins. Phlebology 2009;24:13-33.

3. Clarke-Moloney M, Keane N, O'Connor V, et al. Randomised controlled trial comparing European standard class 1 to class 2 compression stockings for ulcer recurrence and patient compliance. Int Wound $\mathrm{J}$ 2014;11: 404-8.

4. Lie SS, Karlsen B, Ellen Renate Oord ER, et al. Dropout from an eHealth intervention for adults with type 2 diabetes: a qualitative study. J Med Internet Res 2017;19:1-11.

5. Coghlan N, Copley J, Aplin T, Strong J. Patient experience of wearing compression garments post burn injury: a review of the literature. J Burn Care Res 2017;38:260-9.

6. Moffatt, C, Kommala, D, Dourdin, N, Choe, Y. Venous leg ulcers: patient concordance with compression therapy and its impact on healing and prevention of recurrence. Int Wound J 2009;6:386-93.

7. Annells, M, ONeill, J, Flowers, C. Compression bandaging for venous leg ulcers: the essentialness of a willing patient. J Clin Nurs 2008;17:350-9.

8. Field H. Fear of the known? District nurses' practice of compression bandaging. Br J Community Nurs 2004;9:S6S15.

9. Finlayson K, Edwards H, Courtney M. The impact of psychosocial factors on adherence to compression therapy to prevent recurrence of venous leg ulcers. J Clin Nurs 2010;19:1289-97.

10. Harker J. Influences on patient adherence with compression hosiery. J Wound Care 2000;9:379-82.

11. Miller C, Kapp S, Newall N, et al. Predicting concordance with multilayer compression bandaging. J Wound Care 2011;20:101-12.

12. Scheer R. Clinical innovation: compression garments for managing lymphoedema. Wounds Int 2017;8:34-8.

13. Schofield J, Flanagan M, Fletcher J, et al. The provision of leg ulcer services by practice nurses. Nurs Stand 2000;14:54-60.

14. French, L. Community nurse use of Doppler ultrasound in leg ulcer assessment. $\mathrm{Br} \mathrm{J}$ Community Nurs 2005;10:S6-13. 